\title{
Reliability of symmetric dimethylarginine in dogs with myxomatous mitral valve disease as kidney biomarker
}

\author{
Alice Savarese ${ }^{\ddagger}$, , Monica Probo $¥$, Chiara Locatelli, Sergio Aurelio Zanzani, Alessia Libera Gazzonis, Melissa Papa \\ and Paola Giuseppina Brambilla
}

Department of Veterinary Medicine (DIMEVET), University of Milan, via Celoria 10, 20133 Milan, Italy

${ }^{*}$ Both authors contributed equally to this work

\begin{abstract}
The most common cause of heart failure in the canine population is myxomatous mitral valve disease, sometimes complicated by chronic kidney disease. Many studies have been done on the use of symmetric dimethylarginine as biomarker of renal impairment in dogs affected by chronic kidney disease, few studies have examined his reliability as biomarker in dogs affected by heart diseases. Aim of this study was to evaluate symmetric dimethylarginine in dogs affected by mitral valve disease in order to assess his reliability in heart diseases. This was a retrospective case-control study on a clinical population of dogs affected by mitral valve disease (cases) vs healthy dogs (controls). Both groups underwent a complete physical evaluation, echocardiographic examination, complete blood count, biochemical panel, including serum creatinine and urea and urine analysis with protein-to-creatinine ratio. Serum was frozen and sent to IDEXX laboratories for symmetric dimethylarginine determination. General linear model was applied to data. A total number of 24 cases and 7 controls were included. Symmetric dimethylarginine value was in the reference value in the $75 \%(n=18)$ of cases, and in the $43 \%(n=3)$ of controls. Once set symmetric dimethylarginine as dependent variable, no statistical significant differences were found for each variable considered (breed, age, sex, weight, class of cardiomyopathy, presence/absence of valvular disease, presence/absence of congestive heart failure, pharmacological therapy, creatinine and urea concentration). Blood concentration of SDMA resulted not influenced by the variables mentioned above, so it could be considered a reliable marker of early renal impairment in dogs affected by mitral valve disease.
\end{abstract}

Keywords: Biomarker, Cardiovascular-renal disorder, Dog, Myxomatous mitral valve disease, Symmetric dimethylarginine.

\section{Introduction}

The most common acquired heart disease affecting dogs and leading to congestive heart failure (CHF) is myxomatous mitral valve disease (MMVD), also known as endocardiosis, that can reach a prevalence of $100 \%$ in small breed dogs in their old age (Mattin et al., 2015).

The presence of both heart and kidney failure can be defined as cardiorenal syndrome (CRS) (Ronco, 2010; Ronco and Maisel, 2010). In 2015, a Consensus Group was formed to develop definitions and recommendations for CRS approach in veterinary medicine (Pouchelon et al., 2015). The consensus renamed CRS as "Cardiovascular-Renal Disorder" $(\mathrm{CRvD})$ and defined it as a "disease, toxin or druginduced structural and/or functional damage to the kidney and/or cardiovascular system, leading to disruption of the normal interactions between these systems, to the ongoing detriment of one or both" (Pouchelon et al., 2015).

Martinelli et al. (2016), reported a 25\% prevalence of chronic kidney disease (CKD) associated with hyperazotemia in dogs with MMVD. Moreover, the presence of CKD associated to MMVD in $\operatorname{dogs}$ decreases survival time (Martinelli et al., 2016).

An early diagnosis of renal impairment in patients affected by cardiovascular disease is therefore very important. Glomerular filtration rate (GFR) is currently considered the gold standard for quantitative determination of kidney filtration, although not routinely performed on dogs and cats. In fact, the procedure requires the intra-venous injection of a marker of filtration, followed by multiple blood samples at determined time, making the process expensive and time consuming (Relford et al., 2016; Yerramilli et al., 2016).

Currently, the cornerstone in the diagnosis of CKD in veterinary practice is the measurement of serum Creatinine ( $\mathrm{sCr}$ ), that is the most common marker of GFR. Indeed, the use of $\mathrm{sCr}$ has some limitations, since its concentration tend to rise when almost the $75 \%$ of the renal activity is lost and its levels are affected by muscle mass, making the evaluation of renal function more difficult in cachectic and geriatric animals.

*Corresponding Author: Alice Savarese. Department of Veterinary Medicine (DIMEVET), University of Milan, via Celoria 10, 
Many studies have been carried out to assess the efficacy of novel biomarker in the early detection of kidney failure. Due to its characteristics, symmetric dimethylarginine (SDMA) has raised, in the last few years, as the most promising novel biomarker in the early detection of kidney failure. SDMA is one of the methylated forms derived from the arginine metabolism, with asymmetric dimethylarginine (ADMA) and monomethylarginine (MMA) (Kakimoto and Akazawa, 1970). While ADMA is largely cleared by enzymatic hydrolysis, SDMA is primarily eliminated by renal excretion (McDermott, 1976), suggesting its role as endogenous marker of GFR (Kielstein et al., 2006; Schwedhelm and Böger, 2011). SDMA increases earlier than creatinine and can identify a GFR decline as early as a 30\% (Polzin, 2016). A large meta-analysis from Kielstein et al. (2006) showed that SDMA strongly correlated with inulin clearance and sCr. A study from Relford et al. (2016) reported that age and lean body mass cannot influence SDMA concentration in small animals.

Major non-renal systemic disease, including hepatic and endocrine diseases have not been consistently identified as reasons of elevation in serum SDMA concentrations, neither in humans nor in animals, and the effects of both heart and kidney failure on SDMA elimination are still undefined (Schwedhelm and Böger, 2011; Veldink et al., 2013; Bum-Sul et al., 2017).

The aim of this study was to determine the reliability of serum SDMA as a marker of early renal impairment in dogs affected by MMVD in different American College of Veterinary Internal Medicine (ACVIM) classes, and with $\mathrm{sCr}$ within the normal range of values.

\section{Materials and Methods}

This was a retrospective case-control study on a clinical population of dogs affected by MMVD (cases) vs healthy dogs (controls). The animals enrolled belonged to the population of dogs referred to the Cardiology Service of our institution between February 2015 and July 2016.

Inclusion criteria were: diagnosis of MMVD, absence of any other concurrent pathology and no hematological alteration compatible with $\mathrm{CKD}$ ( $\mathrm{sCr}<$ 1,4 mg/dl). Dogs with other heart or systemic diseases were excluded. Control group was composed by healthy dogs that were evaluated at the veterinary teaching hospital for periodic screening and then enrolled. For all dogs, informed consent for every procedure signed by the owner was obtained, as routinely done in our clinical practice.

Both groups underwent a complete physical evaluation, chest $\mathrm{x}$-ray, echocardiographic examination, complete blood count (CBC) and serum biochemical panel. A complete echocardiographic examination was performed in both groups using standardized thoracic imaging planes, according to the recommendations of the American Society of Echocardiography (Thomas et al., 1993). Dogs without any clinical, radiographic and echocardiographic sign of cardiac disease were enrolled as controls. Echocardiography was performed using an Esaote MyLab50 Gold cardiovascular, with phased array multifrequency probes (7.5-10 Mhz and 2.5-3 Mhz).

The investigators performing the echocardiographic exams were all focused on specialty cardiology practice (AS, CL, PGB). Dogs diagnosed with MMVD were subdivided in 4 classes, from $\mathrm{B} 1$ to $\mathrm{D}$, in line with the ACVIM classification. As recommended by the ACVIM guidelines, stage $\mathrm{B}$ included dogs affected by MMVD without signs of CHF (namely, in stage B1, dogs without evidence of cardiac enlargement; in B2, dogs with signs of cardiac remodeling as left atrium (LA) and/or left ventricle (LV) enlargement); stage C included dogs with clinical signs of $\mathrm{CHF}$ due to MMVD, and stage D included subjects presenting both clinical signs of $\mathrm{CHF}$ and refractoriness to standard medical treatments (Atkins et al., 2009; Boswood et al., 2016).

All dogs underwent indirect systemic blood pressure evaluation by Doppler flow meter as reported in ACVIM guidelines (Brown et al., 2007). Based on the International Renal Interest Society (IRIS) guidelines, dogs were classified in 5 classes, from 0 to 4 . No azotemic patients (normal $\mathrm{sCr}$ ) were enrolled, and all the dogs were classified as IRIS class 0 (dogs at risk for the development of CKD). An IRIS substage of CKD based on blood pressure and on the presence of proteinuria was performed, when possible, as reported in the IRIS guidelines (IRIS, 2016).

Blood samples were collected from the cephalic vein during routine clinical evaluation, and $\mathrm{CBC}$ and selected biochemical parameters were performed. For CBC, EDTA was used as anticoagulant, while, for biochemical evaluation, blood was placed in tubes without anticoagulant and centrifuged at $3250 \mathrm{x} \mathrm{g}$ for 5 minutes. CBC was performed using an automated laser hematology analyzer (Sysmex XT-2000iV, Sysmex corporation, Kobe, Japan), while biochemistry was carried out with an automated spectrophotometer (Roche Cobas Mira Classic Biochemistry Analyzer).

The biochemical panel performed was composed by: glucose, urea, sCr, total proteins, sodium, potassium, chloride, alanine transaminase, alkaline phosphatase. An aliquot of serum (at least $300 \mu \mathrm{l}$ ) was frozen and sent to IDEXX laboratories for SDMA determination with the EMIT (Enzyme Multiplied Immunoassay Technology). SDMA in dog was considered normal when $<14 \mu \mathrm{g} / \mathrm{dL}$ (Nabity et al., 2015). Urine were collected via spontaneous micturition or cystocentesis and complete urinalysis with sediment and Urine Protein to Creatinine Ratio (UPC) was performed. 
Normality of data distribution was tested with Shapiro - Wilk test. For baseline descriptive statistics, normally distributed continuous variables were presented as mean and standard deviation, non- normally distributed variables as median and interquartile range (IQR). Data were analyzed with IBM SPSS Statistics 23 [Release 23.0.0.0] using a multivariate generalized linear model (GLM) with backward elimination, applied on ACVIM class (compared with breed, sex, age, weight, SDMA values, Urea values and $\mathrm{sCr}$ values) and on SDMA (compared with breed, sex, age, weight, MVD presence or absence, ACVIM class, presence of clinical symptomatology, pharmacological therapy, Urea values and $\mathrm{sCr}$ values).

To apply the model, a transformation of ACVIM classes into numbers categories was made, with ACVIM class B1 corresponding to 1, ACVIM B2 to 2, ACVIM C to 3 and ACVIM D to 4 . We evaluated if severity of MMVD, CHF symptomatology or pharmacological treatment can influence a single measurement of SDMA levels in dogs affected by the MMVD. $\mathrm{P}<0.05$ was set to indicate statistical significance.

\section{Results}

An overall number of 367 privately owned dogs were admitted to the Cardiology Service of DIMEVET of the University of Milan between February 2015 and July 2016; among these, 148 (40\%) were diagnosed with MMVD. The medical archives were reviewed in order to include only patients with complete medical and laboratory information. Thirty-one dogs were enrolled, 24 in the case group and 7 in the control group.

The median age of dogs enrolled in case group was 11.5 years $( \pm 2.83 \mathrm{SD})$, and the median body weight was 13.8 $\mathrm{kg}( \pm 9.91 \mathrm{SD})$. Most of the dogs included were neutered females $(n=11 ; 46 \%)$, followed by intact males $(n=8 ; 33 \%)$, intact females $(n=3 ; 12 \%)$ and neutered males $(\mathrm{n}=2 ; 9 \%)$. The most represented breeds were Mongrels $(\mathrm{n}=8 ; 33 \%)$, Cavalier King Charles $(\mathrm{n}=2 ; 8 \%)$, Chihuahua $(\mathrm{n}=2 ; 8 \%)$ and Maltese dogs $(\mathrm{n}=2 ; 8 \%)$. Breeds with less than two dogs were grouped and listed as others $(51 \%)$. In this group were also listed Dachshund and Jack Russel terrier.

The median age of the control group was 8.2 years $( \pm 1.92 \mathrm{SD})$, and the median body weight was $21 \mathrm{~kg}$ $( \pm 9.17 \mathrm{SD})$. Most of the controls included were neutered females $(\mathrm{n}=5 ; 71 \%)$, followed by intact males $(n=2 ; 29 \%)$. The most represented breed were mongrels $(\mathrm{n}=3 ; 43 \%)$. Thirteen dogs over 24 were classified as ACVIM B1 (54\%), 8 as ACVIM B2 (33\%) and 3 as ACVIM C (13\%). Symptomatic and non-symptomatic dogs for $\mathrm{CHF}$ were respectively $13 \%(\mathrm{n}=3)$ and $87 \%$ $(\mathrm{n}=21)$. The 3 dogs classified as ACVIM class $\mathrm{C}$ were all under standard triple therapy, composed by furosemide, an ACE inhibitor, and pimobendan.

Renal function was tested measuring serum urea, $\mathrm{sCr}$ and SDMA. sCr was normal in all dogs, as it was an inclusion criteria. Serum urea $(\mathrm{NV}=20-60 \mathrm{mg} / \mathrm{dL})$ was over the reference interval in 8 of the cases $(33 \%)$. None of the controls presented alterations in urea values.

The 25\% ( $n=2)$ of dogs classified as ACVIM B1 presented urea values above the reference interval, so the $24 \%(n=3)$ of ACVIM B2 and the $100 \%$ of ACVIM C. Only medical records of dogs presenting urinalysis were retrospectively included. Urine specific gravity (USG) was normal (NV=1020 - 1060) in $87 \%$ of cases $(21 / 24)$ and in $100 \%$ of the controls for which urinalysis was available. For the case group, 2/3 dogs presented USG lower than reference interval (66\%), while one presented USG over the reference interval (34\%). Both of the dogs with low USG were classified as ACVIM C and were assuming furosemide. No proteinuria was detected in any of the dog considered (UPC < 0.5) (IRIS 2016). SDMA value was normal $(<14 \mu \mathrm{g} / \mathrm{dL})$ in the $75 \%(\mathrm{n}=18)$ of cases, and over the reference interval in the $25 \%(n=6)$. The $31 \%$ of the ACVIM B 1 dogs (4/13) and the $25 \%$ of the ACVIM B2 (2/8) presented SDMA values over the reference interval. None of the dogs classified as ACVIM $\mathrm{C}$ had SDMA above the references. SDMA was above the reference intervals in $4 / 7$ of controls $(57 \%)$

According to the new IRIS guidelines, the $25 \%$ of MMVD dogs should be shifted from IRIS class 0 to IRIS class 1 , while the $57 \%$ of dogs classified as controls switched form IRIS class 0 to IRIS class 1 .

Once set SDMA as dependent variable in the GLM, the results showed no statistical significant difference for each variable considered (breed, age, sex, weight, ACVIM class, healthy/affected, presence of $\mathrm{CHF}$ symptomatology, pharmacological therapy, $\mathrm{sCr}$, urea) (Table 1, Table 2).

Once set ACVIM class as dependent variable in the GLM, the results only showed a statistical significant difference in the variable age for higher ACVIM classes $(\mathrm{C})(\mathrm{P}=0.014 ; \mathrm{OR}=1.3)$ (Table 3, Table 4).

\section{Discussion}

The population of dogs affected by MMVD enrolled in this study presented the typical characteristics reported in literature. In fact, the dogs were mainly of medium age and body weight, mongrels or pure small breed dogs (Borgarelli and Buchanan, 2012). Most of the dogs were asymptomatic for $\mathrm{CHF}(87 \%)$. Age resulted correlated with ACVIM classes, as reported in literature (Borgarelli and Buchanan, 2012). Interestingly, multivariate analysis showed that for each year of life of the dog, the risk of belonging to a higher ACVIM class increases of 1.3 times.

According to literature, the prevalence of azotemia in dogs with heart diseases ranges between 7.4 and $25 \%$. (Atkins et al., 2002; Nicolle et al., 2007; Guglielmini et al., 2013; Martinelli et al., 2016). 
Table 1. Linear generalized univariate model analysis with binomial negative response; dependent variable: SDMA.

\begin{tabular}{|c|c|c|c|c|c|}
\hline \multirow{2}{*}{ Variable } & \multirow{2}{*}{ Factor } & \multirow{2}{*}{ OR } & \multicolumn{2}{|c|}{ IC $95 \%$} & \multirow{2}{*}{ p-value } \\
\hline & & & INF & SUP & \\
\hline \multirow{4}{*}{ Sex } & $\mathrm{F}$ & 1.661 & 0.153 & 18.075 & 0.677 \\
\hline & $\mathrm{FC}$ & 1.072 & 0.166 & 6.931 & 0.942 \\
\hline & $\mathrm{M}$ & 1.397 & 0.212 & 9.200 & 0.728 \\
\hline & MC (reference) & 1 & & & \\
\hline \multirow{2}{*}{ Breed } & Mongrel & 1.036 & 0.357 & 3.012 & 0.948 \\
\hline & Pure (reference) & 1 & & & \\
\hline Age (years) & Continuous Variable & 1.032 & 0.855 & 1.246 & 0.740 \\
\hline Weight $(\mathrm{Kg})$ & Continuous Variable & 0.994 & 0.937 & 1.055 & 0.848 \\
\hline $\mathrm{sCr}(\mathrm{mg} / \mathrm{dL})$ & Continuous Variable & 4.865 & 0.070 & 339.044 & 0.465 \\
\hline Serum urea $(\mathrm{mg} / \mathrm{dL})$ & Continuous Variable & 0.997 & 0.963 & 1.033 & 0.862 \\
\hline ACVIM & Continuous Variable & 0.804 & 0.321 & 2.013 & 0.641 \\
\hline \multirow{2}{*}{ CHF symptoms } & No & 1.241 & 0.052 & 29.905 & 0.894 \\
\hline & Yes (reference) & 1 & & & \\
\hline \multirow{5}{*}{ Pharmacological treatment (CHF) } & ACE-inhibitor & 1.361 & 0.231 & 8.013 & 0.733 \\
\hline & ACE- inhibitor and diuretic & 1.317 & 0.052 & 33.426 & 0.868 \\
\hline & ACE- inhibitor and inotropic agent & 2.219 & 0.025 & 198.372 & 0.728 \\
\hline & ACE- inhibitor, inotropic agent and diuretic & 1.681 & 0.101 & 27.989 & 0.717 \\
\hline & No treatment (reference) & 1 & & & \\
\hline
\end{tabular}

Table 2. Linear generalized multivariate model analysis with binomial negative response [after backward elimination]; dependent variable: SDMA

\begin{tabular}{|c|c|c|c|c|c|}
\hline \multirow{2}{*}{ Variable } & \multirow{2}{*}{ Factor } & \multirow{2}{*}{ OR } & \multicolumn{2}{|c|}{ IC $95 \%$} & \multirow{2}{*}{ p-value } \\
\hline & & & INF & SUP & \\
\hline $\mathrm{sCr}(\mathrm{mg} / \mathrm{dL})$ & Continuous Variable & 2.143 & 0.242 & 19.001 & 0.494 \\
\hline
\end{tabular}

Table 3. Linear generalized univariate model analysis with ordinal logistic response; dependent variable: ACVIM.

\begin{tabular}{lccccc}
\hline \multirow{2}{*}{ Variable } & \multirow{2}{*}{ Factor } & OR & \multicolumn{2}{c}{ IC 95\% } & p-value \\
\cline { 5 - 6 } & & & INF & SUP & 0.265 \\
Sex & F & 10.015 & 0.175 & 573.395 & 0.606 \\
& M & 0.458 & 0.024 & 8.880 & 0.076 \\
Breed & MC (reference) & 15.623 & 0.754 & 323.923 & \\
Age (years) & Mongrel & 1 & & & 0.793 \\
Weight $(\mathrm{Kg})$ & Pure (reference) & 1.270 & 0.214 & 7.542 & \\
sCr $(\mathrm{mg} / \mathrm{dL})$ & Continuous Variable & 1.300 & 0.924 & 1.829 & 0.132 \\
Serum urea $(\mathrm{mg} / \mathrm{dL})$ & Continuous Variable & 0.929 & 0.843 & 1.023 & 0.135 \\
\hline
\end{tabular}

Table 4. Linear generalized multivariate model analysis with ordinal logistic response; dependent variable: ACVIM

\begin{tabular}{|c|c|c|c|c|c|}
\hline \multirow{2}{*}{ Variable } & \multirow{2}{*}{ Factor } & \multirow{2}{*}{ OR } & \multicolumn{2}{|c|}{ IC $95 \%$} & \multirow{2}{*}{ p-value } \\
\hline & & & INF & SUP & \\
\hline Age (years) & Continuous Variable & 1.323 & 1.058 & 1.656 & 0.014 \\
\hline
\end{tabular}


In our population, all dogs presented normal $\mathrm{sCr}$ values. No statistically significant differences in urea values have been found among different ACVIM classes, although a positive increasing trend is evident and the totality of dog in ACVIM class C presented urea values above the reference interval. The progression of MMVD and the development of $\mathrm{CHF}$ reduce renal perfusion and can result in urea increase (Aronson et al., 2004; Kazory, 2010). Also, the activation of the renin angiotensin aldosterone system (RAAS), which increases the absorption of sodium and water, can contribute (Aronson et al., 2004; Kazory 2010). The increase may also be due to the administration of diuretic drugs, prescribed for ACVIM class C patients (Atkins et al., 2009; Pouchelon et al., 2015).

The $75 \%$ of patients at any stage of MMVD presented normal values of SDMA. SDMA has been compared with all the variables considered in our study, and none of them gave statistically significant result. SDMA appears as a promising early biomarker of kidney function in patients affected by any stage of MMVD, since the presence of this cardiovascular disease doesn't result in influences on the parameter. According to literature, our study showed that SDMA is not influenced by lean body mass and weight (Hall et al., 2015), differently from creatinine; therefore, those data cannot be considered as confounding factors. Moreover, SDMA is not influenced by age: this is an important aspect since the canine population affected by MMVD is usually in the old age. Pedersen et al. (2006) reported also that SDMA levels were not altered by the presence of asymptomatic mitral regurgitation, but the study did not consider any dog presenting $\mathrm{CHF}$ and was conducted only on Cavalier King Charles Spaniels dogs (Pedersen et al., 2006).

More recently, Bum-Sul et al. (2017) reported that SDMA levels in dogs with MMVD, mostly symptomatic, were not correlated to age or body weight, but closely correlated to the severity of HF and some echocardiographic markers, like LA/Ao ratio (Bum-Sul et al., 2017). Based on our population, since SDMA is considered a biomarker of GFR (Nabity et al., 2015), our data seems to suggest that the $25 \%$ of our population could present a reduction of GFR despite the normal $\mathrm{sCr}$ values. Even the comparison between SDMA and creatinine didn't result statistically significant, differently from what reported in literature (Nabity et al., 2015), but this data is probably attributable to the inclusion criteria used in this study. In fact, all the patients presented normal creatinine values.

It can be noted that, in the control group, there was a greater percentage of patients with SDMA values higher than normal compared to the group of cases ( $25 \%$ vs $57 \%$ ): this result is of difficult interpretation since, in veterinary medicine, few studies have been made about influences other than CKD on SDMA values and due to the lack of information about abdominal ultrasound. However, all control dogs in our population presented normal urinary specific gravity and no proteinuria, so kidney damage seems less likely. Many different causes have been investigated to justify high levels of SDMA and ADMA concentration in human patients, like in diabetes (Krzyzanowska et al., 2007) or sepsis (Koch et al., 2013), but all the principal diseases that can alter SDMA values have been excluded from our population. We cannot exclude the presence of pre-analytical errors in SDMA determination. Moreover, a recent study reported the presence of a small percentage of animals with elevated SDMA but normal sCR in which kidney function must be assessed (Polzin, 2016).

Although no agreement is present on the therapeutic options in dog presenting alteration of SDMA without other signs of renal impairment, the evaluation of this parameter could be important from a clinical point of view, and allows us to suggest for these patients a more targeted diagnostic procedure with a full assessment of kidney function, including second evaluation of SDMA within one month, complete biochemical renal profile examination (creatinine and urea), urinalysis with UPC ratio and ultrasound renal evaluation, as recommended by IRIS guidelines (IRIS, 2016). Moreover, according to Hall et al. (2017) the administration of a renal diet to dogs currently classified as IRIS-Stage 1 CKD can result in reduction of blood concentration of kidney biomarkers, in particular SDMA and sCr, besides improving patient's quality of life (Hall et al., 2017). A previous longitudinal study on geriatric dogs fed with commercial renal protective food showed that dogs consuming test food showed significant reduction in the concentrations of serum SDMA, sCr, and BUN. Moreover, serum sodium concentrations were seen to be statistically significant lower in dogs assuming this kind of diets, that can be of help in dogs with heart disease in which restriction of sodium is indicated. Finally, a kidney protection food containing more biologically available protein sources with respect to other commercially available dry foods could be of help in preventing cardiac cachexia, loss of muscular masses and mobility in patients affected by MMVD at various gravity levels (Hall et al., 2016). This is a retrospective study and, as such, has some inherent limitations. Firstly, the abdominal ultrasound was not systematically assessed in our population of dogs. Secondly, the small number of animals included in higher ACVIM class $\mathrm{C}$ and the single determination of the parameter could affect the statistical significance and must be taken into account in future studies. In fact, a single determination of SDMA, not followed by a second reevaluation within one month or abdominal imaging, cannot be considered diagnostic of CRvD. 
In conclusion, our study showed that SDMA is free from correlation with breed, age, sex, weight, presence/absence of MMVD, presence of CHF symptoms and pharmacological therapy as well. SDMA can be actually considered a reliable parameter for evaluation of renal function in dogs affected by MMVD, especially in those patients with a nonadvanced stage of disease (ACVIM class B2), for which an early diagnosis of the onset of kidney failure is fundamental in order to plan a diuretic therapy. SDMA repeated measurements over time, as recommended by IRIS guidelines, are necessary (IRIS, 2016), because one determination does not allow us to exclude definitely a later onset of the renal impairment and then to be considered diagnostic in order to highlight a possible onset of CRS.

\section{Conflict of interest}

The authors declare that there is no conflict of interest.

\section{References}

Aronson, D., Mittleman, M.A. and Burger, A.J. 2004. Elevated Blood Urea Nitrogen Level as a Predictor of Mortality in Patients Admitted for Decompensated Heart Failure. Am. J. Med. 116(7), 466-473.

Atkins, C., Bonagura, J., Ettinger, S., Fox, P., Gordon, S., Haggstrom, J., Hamlin, R., Keene, B., LuisFuentes, V. and Stepien, R. 2009. ACVIM Consensus Statement. Guidelines for the diagnosis and treatment of canine chronic valvular heart disease. J. Vet. Intern. Med. 23, 1142-1150.

Atkins, C.E., Brown, W.A., Coats, J.R., Crawford, M.A., DeFrancesco, T.C., Edwards, J., Fox, P.R., Keene, B.W., Lehmkuhl, L., Luethy, M., Meurs, K., Petrie, J.P., Pipers, F., Rosenthal, S., Sidley, J.A. and Straus, J. 2002. Effects of long-term administration of enalapril on clinical indicators of renal function in dogs with compensated mitral regurgitation. J. Am. Vet. Med. Assoc. 221(5), 654658.

Borgarelli, M. and Buchanan, J.W. 2012. Historical review, epidemiology and natural history of degenerative mitral valve disease. J. Vet. Cardiol. 14, 93-101.

Boswood, A., Haggstrom, J., Gordon, S.G., Wess, G., Stepien, R.L., Oyama, M.A., Keene, B.W., Bonagura, J., MacDonald, K.A., Patteson, M., Smith, S., Fox, P.R., Sanderson, K., Woolley, R., Szatmári, V., Menaut, P., Church, W.M., O'Sullivan, M.L., Jaudon, J.P., Kresken, J.G., Rush, J., Barrett, K.A., Rosenthal, S.L., Saunders, A.B., Ljungvall, I., Deinert, M., Bomassi, E., Estrada, A.H., Fernandez Del Palacio, M.J., Moise, N.S., Abbott, J.A., Fujii, Y., Spier, A., Luethy, M.W., Santilli, R.A., Uechi, M., Tidholm, A. and Watson, P. 2016. Effect of Pimobendan in Dogs with
Preclinical Myxomatous Mitral Valve Disease and Cardiomegaly: The EPIC Study - A Randomized Clinical Trial. J. Vet. Intern. Med. 30, 1765-1779.

Brown, S., Atkins, C., Bagley, R., Carr, A., Cowgill, L., Davidson, M., Egner, B., Elliott, J., Henik, R., Labato, M., Littman, M., Polzin, D., Ross, L., Snyder, P. and Stepien, R. 2007. Guidelines for the identification, evaluation, and management of systemic hypertension in dogs and cats. J. Vet. Intern. Med. 21, 542-558.

Bum-Sul, C., Hyeong-Sun, M., Sang-Hyuk, S. and Hyun, C. 2017. Evaluation of serum cystatin-C and symmetric dimethylarginine concentrations in dogs with heart failure from chronic mitral valvular insufficiency. J. Vet. Med. Sci. 79, 41-46.

Guglielmini, C., Poser, H., Pria, A.D., Drigo, M., Mazzotta, E., Berlanda, M. and Luciani, A. 2013., Red blood cell distribution width in dogs with chronic degenerative valvular disease. J. Am. Vet. Med. Assoc. 243, 858-862.

Hall, J.A., Yerramilli, M., Obare, E., Yerramilli, M., Melendez, L,D. and Jewell, D.E. 2015. Relationship between lean body mass and serum renal biomarkers in healthy dogs. J. Vet. Intern. Med. 29, 808-814.

Hall, J.A, MacLeay, J., Yerramilli, M., Obare, E., Yerramilli, M., Schiefelbein, H., Paetau-Robinson, I. and Jewell, D.E. 2016. Positive Impact of Nutritional Interventions on Serum Symmetric Dimethylarginine and Creatinine Concentrations in Client-Owned Geriatric Dogs. PLoS One 11(4), e0153653. doi: 10.1371/journal.pone.0153653.

Hall, J.A., Fritsch, D.A., Yerramilli, M., Obare, E., Yerramilli, M. and Jewell, D.E. 2018. A longitudinal study on the acceptance and effects of a therapeutic renal food in pet dogs with IRIS-Stage 1 chronic kidney disease. J. Anim. Physiol. Anim. Nutr. 102, 297-307.

IRIS Staging for CKD 2016. www.iris-kidney.com.

Kakimoto, Y. and Akazawa, S. 1970. Isolation and identification of N-G, N-G- and N-G, N'-G-80 dimethyl-arginine, N-epsilon-mono-, di-, and trimethyllysine, and glucosylgalactosyl- and galactosyl-delta-hydroxylysine from human urine. J. Biol. Chem. 245, 5751-5758.

Kazory, A. 2010. Emergence of Blood Urea Nitrogen as a Biomarker of Neurohormonal Activation in Heart Failure. Am. J. Cardiol. 106, 694-700.

Kielstein, J.T., Salpeter, S.R. and Bode-Boeger, S.M. 2006. Symmetric dimethylarginine (SDMA) as endogenous marker of renal function-a metaanalysis. Nephrol. Dial. Transplant. 21, 2446-2451.

Koch, A., Weiskirchen, R., Bruensing, J., Dückers, H., Buendgens, L., Kunze, J., Matthes, M., Luedde, T., Trautwein, C. and Tacke, F. 2013. Regulation and Prognostic Relevance of Symmetric 
Dimethylarginine Serum Concentrations in Critical Illness and Sepsis. Mediators Inflamm. 413826.

Krzyzanowska, K., Mittermayer, F. and Shnawa, N. 2007. Asymmetrical dimethylarginine is related to renal function, chronic inflammation and macroangiopathy in patients with type 2 diabetes and albuminuria. Diabetic Med. 24, 81-86.

Martinelli, E., Locatelli, C., Bassis, S., Reis Crosara, S.L., Paltrinieri, S., Scarpa, P., Spalla, I., Zanaboni, A.M., Quintavalla, C. and Brambilla, P.G. 2016. Preliminary Investigation of Cardiovascular-Renal Disorders in Dogs with Chronic Mitral Valve Disease. J. Vet. Intern. Med. 30, 1612-1618.

Mattin, M.J., Boswood, A., Church D.B., LópezAlvarez, J., McGreevy, P.D., O'Neill, D.G., Thomson, P.C. and Brodbelt, D.C. 2015. Prevalence of and risk factors for degenerative mitral valve disease in dogs attending primary-care veterinary practices in England. J. Vet. Intern. Med. 29(3), 847-854.

McDermott, J.R. 1976. Studies on the catabolism of $\mathrm{Ng}$-methylarginine, $\mathrm{Ng}$, $\mathrm{Ng}$-dimethylarginine and $\mathrm{Ng}, \mathrm{Ng}$-dimethylarginine in the rabbit. Biochem. J. 154, 179-184.

Nabity, M.B., Lees, G.E., Boggess, M.M., Yerramilli, M., Obare, E., Yerramilli, M., Rakitin, A., Aguiar, J. and Relford, R. 2015. Symmetric Dimethylarginine Assay Validation, Stability, and Evaluation as a Marker for the Early Detection of Chronic Kidney Disease in Dogs. J. Vet. Intern. Med. 29, 1036-1044.

Nicolle, A.P., Chetboul, V., Allerheiligen, T., Pouchelon, J.L., Gouni, V., Tessier-Vetzel, D., Sampedrano, C.C. and Lefebvre, H.P. 2007. Azotemia and glomerular filtration rate in dogs with chronic valvular disease. J. Vet. Intern. Med. 21, 943-949.

Pedersen, L.G., Tarnow I., Olsen L.H., Teerlink, T. and Pedersen, H.D. 2006. Body size, but neither age nor asymptomatic mitral regurgitation, influences plasma concentrations of dimethylarginines in dogs. Res. Vet. Sci. 80, 336-342.

Polzin, D.J. 2016. SDMA - The Clinician Perspective. In the Proceedings of the 2016 DACVIM ESVNU Pre-congress Symposium, Gothenburg, Sweden.

Pouchelon, J.L., Atkins, C.E., Bussadori, C., Oyama, M,A., Vaden, S.L., Bonagura, J.D., Chetboul, V., Cowgill, L.D., Elliot, J., Francey, T., Grauer, G.F., Fuentes, V.L., Moise, N.S., Polzin, D.J., Van Dongen, A.M. and Van Israël, N. 2015. Cardiovascular-renal axis disorders in the domestic dog and cat: a veterinary consensus statement. J. Small Anim. Pract. 56(9), 537-552.

Relford, R., Robertson, J. and Clements, C. 2016. Symmetric Dimethylarginine: Improving the Diagnosis and Staging of Chronic Kidney Disease in Small Animals. Vet. Clin. North Am. Small Anim. Pract. 46(6), 941-960.

Ronco, C. 2010. Cardiorenal syndromes: definition and classification. Contrib. Nephrol. 164, 33-38.

Ronco, C. and Maisel, A. 2010. Volume overload and cardiorenal syndromes. Congest. Heart Fail. 16(Suppl. 1), Si-iv; quiz Svi.

Schwedhelm, E. and Böger, R.H. 2011. The role of asymmetric and symmetric dimethylarginines in renal disease. Nat. Rev. Nephrol. 7, 275-285.

Thomas, W.P., Gaber, C.E., Jacobs, G.J., Kaplan, P.M., Lombard, C.W., Moise, N.S. and Moses, B.L. 1993. Recommendations for Standards in Transthoracic Two-Dimensional Echocardiography in the Dog and Cat. Journal J. Vet. Intern. Med. 7(4), 247-252.

Veldink, H., Faulhaber, W.R. and Park, J.K. 2013. Effects of chronic SDMA infusion on glomerular filtration rate, blood pressure, myocardial function and renal histology in C57BL6/J mice. Nephrol. Dial. Transplant. 28, 1434-1439.

Yerramilli, M., Farace. G., Quinn, J. and Yerramilli, M. 2016. Kidney Disease and the Nexus of Chronic Kidney Disease and Acute Kidney Injury: The Role of Novel Biomarkers as Early and Accurate Diagnostics. Vet. Clin. North Am. Small Anim. Pract. 46(6), 961-993. 